\title{
REFERENCES
}

1. A. A. Albert and M. S. Frank, Simple Lie algebras of characteristic p, Rend. Sem. Mat. Univ. e Politech. di Torino vol. 14 (1955) pp. 117-139.

2. Richard Block, New simple Lie algebras of prime characteristic, Trans. Amer. Math. Soc. vol. 89 (1958) pp. 421-449.

3. S. A. Jennings and Rimhak Ree, On a family of Lie algebras of characteristic $p$, Trans. Amer. Math. Soc. vol. 84 (1957) pp. 192-207.

The University of British Columbia, Vancouver, B. C.

\section{A NOTE ON COMPLETE BOOLEAN ALGEBRAS}

\section{R. S. PIERCE}

1. Introduction. Among commutative rings, Boolean algebras stand just below fields in simplicity of structure. In contrast, little is known concerning their classification. The purpose of this paper is to present a decomposition theorem for complete Boolean algebras, which, in a small way, simplifies the classification problem. As an illustration of how this decomposition theorem can be used, it is shown that an infinite cardinal number $\boldsymbol{N}$ can be the power of a complete Boolean algebra if and only if $\boldsymbol{\aleph}^{\boldsymbol{N}_{0}}=\boldsymbol{N}$.

The words "complete Boolean algebra" will henceforth often be abbreviated C.B.A. Similarly, B.A. stands for Boolean algebra. The join, meet and complement operations in a B.A. are denoted $V$, $\wedge$ and $\left({ }^{\prime}\right)$. Inclusion is indicated by $\leqq$. Also 0 and $u$ respectively stand for the zero and unit elements of a B.A. The least upper bound of a subset $A$ of a C.B.A. is designated l.u.b. $A$.

The relation of isomorphism between Boolean algebras is denoted $\cong$. If $B$ is a B.A. and $a \in B$, then $B_{a}$ will stand for the set $\{b \in B \mid b \leqq a\}$. If $a \neq 0$, then $B_{a}$ is a B.A. (which is complete if $B$ is complete) with the join, meet and zero inherited from $B$ and with complementation relative to $a$. That is, the complement of $b \leqq a$ in $B_{a}$ is $b^{\prime} \wedge a$. A Boolean algebra $B$ is called homogeneous if $B_{a} \cong B$ for all $a \neq 0$ in $B$. A useful property of C.B.A.'s is they satisfy the Schroeder-Bernstein theorem: if $B \cong \bar{B}_{a}$ and $\bar{B} \cong B_{a}(a \in B, \bar{a} \in \bar{B})$, then $B \cong \bar{B}$. This fact is proved in various places, perhaps the most accessible of which is [5, Theorem 1.31].

The direct union of a set $\left\{B_{\sigma} \mid \sigma \in S\right\}$ of B.A.'s is defined in the usual way (see $[1, \mathrm{p}$. viii $]$ ). This direct union will be denoted $\sum_{\sigma \in S} B_{\sigma}$. There is a useful internal characterization of direct unions of C.B.A.'s.

Received by the editors May 2, 1958. 
Lemma 1.1. If $B$ is a C.B.A., then $B \cong \sum_{\sigma \in S} B_{\sigma}$ if and only if there exists a subset $A=\left\{a_{\sigma} \mid \sigma \in S\right\}$ of pairwise disjoint, nonzero elements of $B$ such that 1.u.b. $A=u$ and $B_{\sigma} \cong B_{a_{\sigma}}$ for all $\sigma \in S$.

The proof comes directly from the definition of direct union, using the completeness of $B$. This lemma suggests the notation $B=\sum_{a \in A} B_{a}$, when $A$ is a set of pairwise disjoint nonzero elements of $B$ and l.u.b. $A$ $=u$.

2. The decomposition theorem. The technique presented in this section is not new. For example, a particular case of it is used in Maharam's paper [4]. Nevertheless, it seems worthwhile to put the idea in a general setting. ${ }^{1}$

Definition 2.1. A cardinal property $\nu$ of complete Boolean algebras is a rule which assigns to each complete Boolean algebra $B$ a unique cardinal number $\nu(B)$, such that if $B \cong \bar{B}$, then $\nu(B)=\nu(\bar{B})$.

The cardinal property is called monotone on $B$ if $\nu\left(B_{a}\right) \leqq \nu\left(B_{b}\right)$ whenever $0 \neq a \leqq b$ in $B$.

A complete Boolean algebra $B$ is called $\nu$-homogeneous if $\nu\left(B_{a}\right)$ $=\nu(B)$ for all $a \neq 0$ in $B$.

Of particular interest are those cardinal properties which are monotone on every C.B.A. All of the following examples enjoy this distinction.

Examples 2.2. (i) $\kappa(B)=$ cardinality of $B$. (ii) $\gamma(B)=$ least cardinal number of a dense subset of $B-\{0\}$. That is, $\gamma(B)$ is the least cardinal $\aleph$ for which there exists a subset $A$ of $B-\{0\}$ with the properties $\kappa(A)=\boldsymbol{N}$ and for any $b \in B-\{0\}$ there exists $a \in A$ such that $a \leqq b$. (iii) $\delta(B)=$ least cardinal $\aleph$ such that if $A \subseteq B$ is a set of nonzero pairwise disjoint elements, then $\kappa(A) \leqq \boldsymbol{N}$.

Note that the above cardinal properties can also apply to arbitrary subsets of a B.A. (and in the case of $\kappa$, to arbitrary sets). For instance, $\delta(T)$ is the least cardinal number $\geqq$ the power of every disjointed (i.e., pairwise disjoint) subset of $T-\{0\}$.

TheOREM 2.3. Let $\nu$ be a cardinal property which is monotone on the complete Boolean algebra $B$. Then $B$ decomposes uniquely in the form

(i) $B=\sum_{a \in A} B_{a}$, where

(ii) $B_{a} \cong \sum_{\sigma \in S_{a}} B_{a \sigma}$ (not generally unique), with each $B_{a \sigma}$ a $\nu$-homogeneous C.B.A. such that $\nu\left(B_{a \sigma}\right)=\boldsymbol{\aleph}_{a}$ and $\boldsymbol{\aleph}_{a} \neq \boldsymbol{\aleph}_{b}$ for $a \neq b$.

1 This method has also been used in a more general setting by P. Erdös and A. Tarski (Ann. of Math. vol. 44 (1943) pp. 315-329). 
Proof. Let $a_{\aleph}=\left(1 . u . b . \quad\left\{b \mid \nu\left(B_{b}\right) \leqq \boldsymbol{N}\right\}\right) \wedge\left(\text { l.u.b. } \quad\left\{b \mid \nu\left(B_{b}\right)<\boldsymbol{N}\right\}\right)^{\prime}$. Take $A$ to be the set of all nonzero $a_{\aleph}$. Clearly $A$ is a set of pairwise disjoint elements of $B$. If 1.u.b. $A<u$, let $0 \neq c=(\text { l.u.b. } A)^{\prime}$. Define $\boldsymbol{N}=\min \left\{\nu\left(B_{b}\right) \mid 0 \neq b \leqq c\right\}$. Then $0 \neq b \leqq c$ and $\nu\left(B_{b}\right)=\boldsymbol{N}$ for some $b$. Also, $0 \neq d \leqq b$ implies $\nu(d) \geqq \boldsymbol{N}$. Hence $b \leqq a_{\aleph} \leqq$ l.u.b. $A$, contrary to (1.u.b. $A) \wedge c=0$. Thus, l.u.b. $A=u$. By $1.1, B=\sum_{a \in A} B_{a}$.

For each $\aleph$ such that $a_{\aleph} \neq 0$, let $A_{\aleph}$ be a maximal set of nonzero, pairwise disjoint elements $b \leqq a_{\mathbb{N}}$ satisfying $\nu\left(B_{b}\right)=\boldsymbol{N}$. Then

$$
\text { l.u.b. } A_{\aleph}=a_{\aleph} \text {. }
$$

Indeed, if $0 \neq c=a_{\aleph} \wedge$ (1.u.b. $\left.A_{\aleph}\right)^{\prime}$, then $d=c \wedge b \neq 0$ for some $b$ satisfying $\nu\left(B_{b}\right) \leqq \boldsymbol{N}$. Hence, by monotonicity, $\nu\left(B_{d}\right) \leqq \nu\left(B_{b}\right) \leqq \boldsymbol{N}$. On the other hand, $d \leqq a_{\aleph} \leqq\left(\text { l.u.b. }\left\{b \mid \nu\left(B_{b}\right)<\boldsymbol{N}\right\}\right)^{\prime}$ implies $\nu\left(B_{d}\right) \geqq \boldsymbol{N}$. Thus, $\nu\left(B_{d}\right)=\boldsymbol{\aleph}$ and the maximality of $A_{\aleph}$ is contradicted. This proves $B_{a_{\aleph}}=\sum_{b \in A_{\aleph}} B_{b}$. Moreover, each $B_{b}\left(b \in A_{\aleph}\right)$ is $\nu$-homogeneous with $\nu\left(B_{b}\right)=\boldsymbol{\aleph}$, since, as was just noted, $0 \neq d \leqq b \in A_{\aleph}$ implies

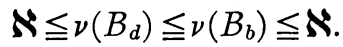

It remains to observe that the decomposition (i) is unique. This is clear since, subject to condition (ii), the unit $a$ of $B_{a}$ is the join of elements $b$ such that $\nu\left(B_{b}\right)=\boldsymbol{\aleph}_{a}$. On the other hand, if $0 \neq c \leqq a$, then $b \wedge c \neq 0$ for some $b$ such that $B_{b}$ is $\nu$-homogeneous with $\nu\left(B_{b}\right)=\boldsymbol{\aleph}_{a}$. Therefore $\boldsymbol{\aleph}_{a}=\nu\left(B_{b \wedge c}\right) \leqq \nu\left(B_{c}\right)$. Consequently $a=a_{\aleph_{a}}$.

3. The power of complete Boolean algebras. Professor B. Jónsson has proposed the following question: what are the possible cardinalities of complete, homogeneous Boolean algebras? In this section Jónsson's question will be answered. ${ }^{2}$ Moreover, by using 2.3 , it will be possible to determine the cardinalities of arbitrary complete Boolean algebras.

First note that any finite B.A. is complete. It is well known (see $\left[1\right.$, p. 159]) that any finite B.A. has $2^{n}$ elements for some integer $n$. Thus, only infinite C.B.A.'s are to be considered.

Theorem 3.1. If $B$ is an infinite C.B.A. of cardinality $\boldsymbol{N}$, then $\aleph^{N} N_{0}=\aleph$.

Proof. By $2.3, B \cong \sum_{\sigma \in S} B_{\sigma}$, where each $B_{\sigma}$ is $k$-homogeneous. In particular, if $B_{\sigma}$ is finite, then $\kappa\left(B_{\sigma}\right)=2$. Let $S=S^{\prime} \cup S^{\prime \prime}$, where $S^{\prime}$ consists of all $\sigma$ such that $\kappa\left(B_{\sigma}\right)=2$ and $S^{\prime \prime}$ are those $\sigma$ for which $B_{\sigma}$ is infinite. Then clearly $\boldsymbol{N}=\kappa(B)=2^{\kappa\left(S^{\prime}\right)}$. $\prod_{\sigma \in S^{\prime \prime}} \kappa\left(B_{\sigma}\right)$. If $\kappa\left(S^{\prime}\right)$ is finite, then (since $\boldsymbol{N}$ is infinite) $2^{\kappa\left(S^{\prime}\right)} \cdot \prod_{\sigma \in S^{\prime \prime}} \kappa\left(B_{\sigma}\right)=\prod_{\sigma \in S^{\prime \prime}} \kappa\left(B_{0}\right)$

${ }^{2}$ Seymour Ginsburg (Proc. Amer. Math. Soc. vol. 9 (1958) pp. 130-132) has shown that complete homogeneous Boolean algebras of power $2^{\mathbf{N}}$ exist for any infinite cardinal $k$. 
and $\boldsymbol{\aleph}^{\boldsymbol{N}_{0}}=\prod_{\sigma \in S^{\prime \prime}} \kappa\left(B_{0}\right)^{\boldsymbol{N}_{0}}$. If $\kappa\left(S^{\prime}\right)$ is infinite, $\boldsymbol{\aleph}^{\boldsymbol{N}_{0}}=2^{\boldsymbol{N}_{0} \cdot \kappa\left(S^{\prime}\right)}$

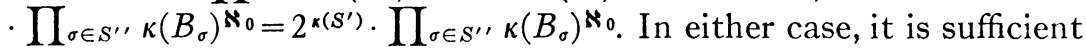
to prove: if $B_{\sigma}$ is an infinite, $\kappa$-homogeneous C.B.A., then $\kappa\left(B_{\sigma}\right)^{N_{0}}$ $=\kappa\left(B_{\sigma}\right)$.

If $B_{\sigma}$ is an infinite C.B.A., then clearly $\delta\left(B_{\sigma}\right) \geqq \boldsymbol{\aleph}_{0}$. Hence $B_{\sigma}$ $=\sum_{i=1}^{\infty} B_{i}$, where $B_{i}=B_{a_{i}}$ for some $a_{i} \neq 0$ in $B$. If $B_{\sigma}$ is also $\kappa$-homogeneous, then $\kappa\left(B_{\sigma}\right)=\kappa\left(B_{i}\right)$ for all $i$. Hence, $\kappa\left(B_{\sigma}\right)=\prod_{i=1}^{\infty} \kappa\left(B_{i}\right)$ $=\kappa\left(B_{\sigma}\right)^{\aleph_{0}}$. This proves the theorem. The following lemma, relating the cardinal properties $\kappa, \gamma$ and $\delta$ of 2.2, prepares the way for the converse of 3.1 .

Lemma 3.2. Let $B$ be a B.A. and $T$ a dense subset of $B$ such that (i) $\kappa(T)=\boldsymbol{\aleph}_{\alpha}$, (ii) $\delta(T)=\boldsymbol{\aleph}_{\beta}$, (iii) $\left(\boldsymbol{\aleph}_{\alpha}\right) \boldsymbol{\aleph}_{\beta}=\boldsymbol{\aleph}_{\alpha}$. Then $\kappa(B)=\boldsymbol{\aleph}_{\alpha}$.

Proof. Since $T$ is dense in $B$, every nonzero element of $B$ is the least upper bound of some disjointed subset of $T$. Indeed, if $b \neq 0$ in $B$ and $X$ is a maximal disjoined subset of $T$ with $x \leqq b$ for all $x \in X$, then l.u.b. $X=b$. For otherwise, there exists $c \neq 0$ in $B$ with $c \leqq b$ and $c \wedge x=0$ for all $x \in X$. Choosing $t \in T$ such that $0 \neq t \leqq c$ gives $X \cup\{t\}$ -a disjointed subset of $T$ composed of elements $\leqq b$. Since $c \wedge x=0$ for all $x \in X$, it cannot happen that $t \in X$. Therefore $X \cup\{t\}$ properly contains $X$. But this contradicts the maximality of $X$. Thus, l.u.b. $X=b$, as claimed. Since $\delta(T)=\boldsymbol{\aleph}_{\beta}$, it follows that every element of $B$ is a join of some subset $X$ of $T$ with $\kappa(X) \leqq \boldsymbol{\aleph}_{\beta}$. Thus $\boldsymbol{\aleph}_{\alpha}=\kappa(T)$ $\leqq \kappa(B) \leqq\left(\boldsymbol{\aleph}_{\alpha}\right) \aleph_{\beta}=\boldsymbol{\aleph}_{\alpha}$.

THEOREM 3.3. If $\boldsymbol{\aleph}$ is an infinite cardinal number satisfying $\boldsymbol{\aleph}_{0}=\boldsymbol{\aleph}$, then there is a complete, homogeneous Boolean algebra of power $\mathbf{\aleph}$.

Proof. Let $S$ be a set of cardinality $\aleph$. For $\sigma \in S$, denote $X_{\sigma}$ $=\left\{x_{\sigma 1}, x_{\sigma 2}\right\}$, the two element discrete space. Let $X=\prod_{\sigma \in S} X_{\sigma}$ be the cartesian product space. Then $X$ is a totally disconnected, compact Hausdorff space. Let $B$ be the C.B.A. of regular open sets of $X$ (see [1, p. 177]). Let $T$ be all subsets of $X$ which are of the form:

$$
Y=\bigcap_{\sigma \in F} \pi_{\sigma}^{-1}\left(\left\{x_{\sigma f(\sigma)}\right\}\right)
$$

where $F$ is a finite subset of $X, f \in\{1,2\}$ and $\pi_{\sigma}: X \rightarrow X_{\sigma}$ is the usual component projection. These sets are clearly open-and-closed (hence regular open) and form a basis for the topology of $X$. Thus, $T$ is a dense subset of $B$. Also, as a topological space, each $Y$ is a cartesian product of $\boldsymbol{\aleph} 2$-point spaces, thus homeomorphic to $X$. It follows that $B \cong B_{Y}$ for all such $Y$. Since $T$ is dense, the Schroeder-Bernstein 
theorem implies that $B$ is homogeneous. It is clear that $\kappa(T)$ $=\boldsymbol{N}+\boldsymbol{N}^{2}+\cdots=\boldsymbol{N}$. Hence, according to 3.2 , the proof is completed by the observation $\delta(T)=\boldsymbol{\aleph}_{0}$. The proof of this fact is sketched in $[3$, p. 166].

REMARK. The C.B.A. constructed in 3.3 is precisely the completion by cuts of the free B.A. with $\boldsymbol{\aleph}$ generators.

Combining Theorems 3.1 and 3.3 wins the objective of this section: an infinite cardinal $\boldsymbol{\aleph}$ can be the power of a complete, or complete homogeneous Boolean algebra if and only if $\boldsymbol{N}^{\boldsymbol{N}_{0}}=\boldsymbol{\aleph}$. One may ask what cardinals satisfy $\boldsymbol{N}_{0}=\boldsymbol{N}$. This equality prevails if $\boldsymbol{\aleph}$ is of the form $2 \boldsymbol{N}^{\prime}$ for some infinite $\boldsymbol{\aleph}^{\prime}$. It does not hold if $\boldsymbol{\aleph}$ is a countable sum of smaller cardinal numbers (by König's theorem-see [2, p. 34]). Little more can be said with certainty. But if the generalized continuum hypothesis is assumed, the situation becomes clearer: $\left(\boldsymbol{\aleph}_{\alpha}\right)^{\boldsymbol{N}_{0}}$ $=\boldsymbol{\aleph}_{\alpha}$ if $\alpha$ is not a limit ordinal, or if $\alpha$ is a limit ordinal which (considered as a well ordered set) contains no countable, cofinal subset (see [6, p. 9, Theorem 7]); otherwise $\left(\boldsymbol{\aleph}_{\alpha}\right)^{\boldsymbol{\aleph}_{0}}>\boldsymbol{\aleph}_{\alpha}$.

\section{REFERENCES}

1. G. Birkhoff, Lattice theory, Amer. Math. Soc. Colloquium Publications, vol. 25 2d ed., 1948.

2. F. Hausdorf, Mengenlehre, 3d ed., Berlin, 1935.

3. J. L. Kelley, General topology, New York, 1955.

4. D. Maharam, On homogeneous measure algebras, Proc. Nat. Acad. Sci. U.S.A. vol. 28 (1942) pp. 108-111.

5. A. Tarski, Cardinal algebras, New York, 1948.

6. - , Quelques théorèmes sur les alephs, Fund. Math. vol. 7 (1925) pp. 1-14.

UNIVERSITY OF WASHINGTON 